\title{
Front Matter: Volume 6475
}

, "Front Matter: Volume 6475," Proc. SPIE 6475, Integrated Optics: Devices, Materials, and Technologies XI, 647501 (13 March 2007); doi:

$10.1117 / 12.727285$

SPIE Event: Integrated Optoelectronic Devices 2007, 2007, San Jose, California, SPIE. United States 


\title{
PROCEEDINGS OF SPIE
}

\section{Integrated Optics: Devices, Materials, and Technologies XI}

\author{
Yakov Sidorin \\ Christoph A. Waechter \\ Editors
}

22-24 January 2007

San Jose, California, USA

Sponsored and Published by

SPIE-The International Society for Optical Engineering

Volume 6475

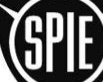

The International Society for Optical Engineering

Proceedings of SPIE-The International Society for Optical Engineering, 9780819465887, v. 6475

SPIE is an international technical society dedicated to advancing engineering and scientific applications of optical, photonic, imaging, electronic, and optoelectronic technologies. 
The papers included in this volume were part of the technical conference cited on the cover and title page. Papers were selected and subject to review by the editors and conference program committee. Some conference presentations may not be available for publication. The papers published in these proceedings reflect the work and thoughts of the authors and are published herein as submitted. The publisher is not responsible for the validity of the information or for any outcomes resulting from reliance thereon.

Please use the following format to cite material from this book:

Author(s), "Title of Paper," in Integrated Optics: Devices, Materials, and Technologies XI, edited by Yakov Sidorin, Christoph A. Waechter, Proceedings of SPIE Vol. 6475 (SPIE, Bellingham, WA, 2007) Article CID Number.

ISSN 0277-786X

ISBN 9780819465887

Published by

SPIE-The International Society for Optical Engineering

P.O. Box 10, Bellingham, Washington 98227-0010 USA

Telephone 1 360/676-3290 (Pacific Time) · Fax 1 360/647-1445

http://www.spie.org

Copyright (C) 2007, The Society of Photo-Optical Instrumentation Engineers

Copying of material in this book for internal or personal use, or for the internal or personal use of specific clients, beyond the fair use provisions granted by the U.S. Copyright Law is authorized by SPIE subject to payment of copying fees. The Transactional Reporting Service base fee for this volume is $\$ 18.00$ per article (or portion thereof), which should be paid directly to the Copyright Clearance Center (CCC), 222 Rosewood Drive, Danvers, MA 01923. Payment may also be made electronically through CCC Online at http://www.copyright.com. Other copying for republication, resale, advertising or promotion, or any form of systematic or multiple reproduction of any material in this book is prohibited except with permission in writing from the publisher. The CCC fee code is 0277 $786 \times / 07 / \$ 18.00$

Printed in the United States of America. 


\section{Contents}

ix Conference Committee

\section{SESSION 1 TUNABLE WGS AND WG SENSORS}

647502 Characterization of quality-factor tunable integrated silicon microtoroidal resonators [6475-01]

J. Yao, D. Levenberger, M. C. WU, Univ. of California, Berkeley (USA)

647503 Micro-photonic cylindrical waveguide based protein biosensor [6475-02]

S. K. Padigi, K. Aasante, R. K. K. Reddy, V. S. R. Kovvuri, A. LaRosa, S. Prasad, Portland State Univ. (USA)

647504 Optical waveguide biosensor based on two-dimensional diffractive elements obtained by nano-imprint lithography [6475-03]

S. Grego, RTI International (USA); Y. Cao, Univ. of North Carolina, Charlotte (USA):

C. A. Bower, B. R. Stoner, RTI International (USA); T. J. Suleski, Univ. of North Carolina,

Charlotte (USA)

647505 Demonstration of a liquid core optical ring resonator sensor coupled with an ARROW waveguide array [6475-04]

I. M. White, H. Oveys, X. Fan, Univ. of Missouri, Columbia (USA); T. L. Smith, J. Zhang, 3M Co. (USA)

\section{SESSION 2 GLASS WG OPTICS}

647507 Glass integrated optics: state of the art and position toward other technologies (Invited Paper) [6475-06]

J.-E. Broquin, Institut de Microélectronique, Electromagnétisme et Photonique, INP Grenoble (France)

Pagination: Proceedings of SPIE follow an e-First publication model, with papers published first online and then in print and on CD-ROM. Papers are published as they are submitted and meet publication criteria. A unique, consistent, permanent citation identifier (CID) number is assigned to each article at the time of the first publication. Utilization of CIDs allows articles to be fully citable as soon they are published online, and connects the same identifier to all online, print, and electronic versions of the publication.

SPIE uses a six-digit CID article numbering system in which:

- The first four digits correspond to the SPIE volume number.

- The last two digits indicate publication order within the volume using a Base 36 numbering system employing both numerals and letters. These two-number sets start with 00, 01, 02, 03, 04, 05, 06, 07, 08, 09, 0A, OB ... 0Z, followed by 10-1Z, 20-2Z, etc.

The CID number appears on each page of the manuscript. The complete citation is used on the first page, and an abbreviated version on subsequent pages. 
647508 Chalcogenide waveguide for IR optical range [6475-07]

M. L. Anne, V. Nazabal, V. Moizan, C. Boussard-Pledel, B. Bureau, J. L. Adam, Equipe Verres et Céramiques, CNRS, Univ. Rennes I (France); P. Nemec, M. Frumar, Univ. of Pardubice (Czech Republic); A. Moreac, Groupe Matière condensée et Matériaux, CNRS, Univ. Rennes I (France); H. Lhermite, IETR-Microelectronique, Univ. Rennesl (France); P. Camy, J. L. Doualan, CNRS-CEA-ENSICAEN, Univ. Caen (France); J. P. Guin, LARMAUER, CNRS, Univ. Rennes 1 (France); J. Le Person, F. Colas, C. Compere, M. Lehaitre, IFREMER (France); F. Henrio, D. Bosc, J. Charrier, École Nationale Supérieure des Sciences Appliquées et de Technologie (France); A.-M. Jurdyc, B. Jacquier, LPCML, Univ. de Lyon I (France)

647509 Channel waveguides fabrication in $\mathrm{Er}^{3+}$-doped tellurite glass by ion beam irradiation [6475-08]

S. Berneschi, Istituto di Fisica Applicata Nello Carrara-CNR (Italy) and Univ. of Bologna (Italy); M. Brenci, Istituto di Fisica Applicata Nello Carrara-CNR (Italy); G. Nunzi Conti, Enrico Fermi Ctr. for Study and Research (Italy) and Istituto di Fisica Applicata Nello Carrara-CNR (Italy); S. Pelli, G. C. Righini, Istituto di Fisica Applicata Nello Carrara-CNR (Italy); I. Bányász, A. Watterich, Research Institute for Solid State Physics and Optics (Hungary); N. Q. Khanh, M. Fried, Research Institute for Technical Physics and Materials Science (Hungary); F. Pászti, Research Institute for Particle and Nuclear Physics (Hungary)

64750 A Three-dimensional integration of passive functions on glass by means of selectively buried waveguides and multiple ion-exchanges [6475-09]

J. Grelin, D. Bucci, E. Ghibaudo, J.-E. Broquin, Institut de Microélectronique,

Electromagnétisme et Photonique, INP Grenoble (France)

SESSION 3 SUBWAVELENGTH DIFFRACTIVE PHOTONICS

64750B A reconfigurable self-collimation-based photonic crystal switch in silicon (Invited Paper) [6475-10]

R. Martin, A. Sharkawy, C. Chen, B. Miao, E. J. Kelmelis, D. W. Prather, EM Photonics (USA)

64750C Distributed subwavelength grating demultiplexer in SOI [6475-11]

E. Bisaillon, McGill Univ. (Canada); D. T. H. Tan, Univ. of British Columbia (Canada);

M.-C. Nadeau, McGill Univ. (Canada); L. Chrostowski, Univ. of British Columbia (Canada);

A. Kirk, McGill Univ. (Canada)

$64750 \mathrm{E}$ Narrowband Bragg reflectors in Ti:LiNbO ${ }_{3}$ optical waveguides and implantation to optical modulators [6475-13]

R. Kim, Advanced Micro Devices (USA); J. Zhang, 3M Corp. Research Materials Lab. (USA);

O. Eknoyan, H. F. Taylor, Texas A\&M Univ. (USA); T. L. Smith, 3M Corp. Research Materials Lab. (USA)

$64750 \mathrm{~F}$ The quarter-wave Bragg reflection waveguide: analytical solutions and properties [6475-14]

B. R. West, McGill Univ. (Canada); A. Helmy, Univ. of Toronto (Canada)

SESSION 4 MODELING

$64750 \mathrm{~S}$ Self-focusing in high-power optical fibers (Invited Paper) [6475-15]

G. R. Hadley, A. V. Smith, Sandia National Labs. (USA) 
$64750 \mathrm{H}$ Goal oriented adaptive finite element method for precise simulation of optical components [6475-16]

L. Zschiedrich, S. Burger, J. Pomplun, F. Schmidt, Zuse Institute Berlin (Germany) and JCMwave GmbH (Germany)

64750I Design issues with MMI-based photonics switches and routers [6475-17]

L. W. Cahill, La Trobe Univ. (Australia)

64750J Light extraction from OLEDs: the waveguide perspective [6475-18]

N. Danz, D. Michaelis, C. Wächter, Fraunhofer-Institut für Angewandte Optik und

Feinmechanik (Germany)

64750K Theoretical analysis of active ring microresonator filter [6475-19]

H. Chen, Yangtze Univ. (China) and College of Optical Sciences, Univ. of Arizona (USA)

64750L Automatic design and optimisation of Si nanophotonics devices using finite element frequency domain solvers [6475-20]

T. P. Felici, D. F. G. Gallagher, L. Bolla, Photon Design (United Kingdom)

\section{SESSION $5 \quad$ RECENT ADVANCES}

64750M Closed-loop design of a semiconductor laser (Invited Paper) [6475-21]

J. V. Moloney, J. Hader, Nonlinear Control Strategies Inc. (USA), College of Optical Sciences, Univ. of Arizona (USA), and Univ. of Arizona (USA); L. Fan, M. Fallahi, College of Optical Sciences, Univ. of Arizona (USA); S. W. Koch, W. Stolz, Univ. of Marburg (Germany)

64750N Integrated optoelectronics in an optical fiber (Invited Paper) [6475-22]

J. V. Badding, Pennsylvania State Univ. (USA); P. J. Sazio, Univ. of Southampton (United Kingdom); V. Gopalan, Pennsylvania State Univ. (USA); A. Amezcua Correa, Univ. of Southampton (United Kingdom); T. J. Scheidemantel, Pennsylvania State Univ. (USA); C. E. Finlayson, Univ. of Southampton (United Kingdom); N. F. Baril, B. R. Jackson, D. Wong, Pennsylvania State Univ. (USA)

64750P A new physical approach to understanding bend loss in optical fibers (Invited Paper) [6475-24]

J. D. Love, C. Durniak, The Australian National Univ. (Australia)

SESSION 6 PLASMONICS

64750Q Design of mid-infrared photodetectors enhanced by surface plasmons on grating structures [6475-25]

Z. YU, G. Veronis, M. L. Brongersma, S. Fan, Stanford Univ. (USA)

64750R SPR waveguide sensor based on combined sensing of phase and amplitude changes [6475-26]

R. Levy, S. Ruschin, Tel-Aviv Univ. (Israel)

64750S Compact couplers between dielectric and plasmonic slot waveguides [6475-27]

G. Veronis, S. Fan, Stanford Univ. (USA) 
$64750 \mathrm{U}$ Long-range surface plasmon waveguides and devices in lithium niobate: preliminary results [6475-29]

P. Berini, Univ. of Ottawa (Canada) and Spectalis Corp. (Canada); G. Mattiussi, N. Lahoud,

R. Charbonneau, Spectalis Corp. (Canada)

\section{SESSION 7 SUBWAVELENGTH AND MICROSTRUCTURES}

$64750 \mathrm{~V}$ Digital holographic microscopy for nanometric quality control of micro-optical components [6475-31]

J. Kühn, F. Charrière, École Polytechnique Fédérale de Lausanne (Switzerland); T. Colomb, École Polytechnique Fédérale de Lausanne (Switzerland) and DP-CHUV (Switzerland); E. Cuche, Y. Emery, Lyncée Tec SA (Switzerland); C. Depeursinge, École Polytechnique Fédérale de Lausanne (Switzerland)

64750X Two-photon absorption for the realization of optical waveguides on printed circuit boards [6475-34]

G. Langer, M. Riester, Austria Technologie \& Systemtechnik (Austria)

64750Y Micro ring cavity resonator incorporating total internal reflection mirrors [6475-35]

D. G. Kim, W. K. Choi, Y. W. Choi, Chung-Ang Univ. (South Korea); J. C. Yi, Hong-lk Univ. (South Korea); Y. Chung, Kwangwoon Univ. (South Korea); N. Dagli, Univ. of California, Santa Barbara (USA)

\section{SESSION $8 \quad$ PHC-BASED DEVICES}

$64750 Z$ Nanophotonic integrated lasers (Invited Paper) [6475-36]

M. Kamp, H. Scherer, Univ. Würzburg (Germany); K. Janiak, H. Heidrich, Fraunhofer HHI (Germany); R. Brenot, G.-H. Duan, Alcatel Thales III-V Lab. (France); H. Benisty, Institut d'Optique-LCFIO (France); A. Forchel, Univ. Würzburg (Germany)

647510 Superprism phenomena in 2D low index contrast polymer photonic crystal [6475-37] L. Wang, Univ. of Texas at Austin (USA); W. Jiang, Omega Optics Inc. (USA); X. Chen, J. Chen, S. Zhang, R. Chen, Univ. of Texas at Austin (USA)

647511 Silicon-based low-loss photonic crystal waveguides [6475-38]

D. Pergande, Martin Luther Univ. Halle-Wittenberg (Germany) and Univ. of Paderborn (Germany); A. Milenin, Max-Planck-Institute of Microstructure Physics (Germany); T. Geppert, Max-Planck-Institute of Microstructure Physics (Germany) and Univ. Paderborn (Germany); A. von Rhein, Univ. of Paderborn (Germany); R. B. Wehrspohn, Martin-LutherUniv. Halle-Wittenberg (Germany) and Univ. of Paderborn (Germany)

647512 Photonic crystal slab reflectors for compact passive and active optical devices [6475-39] S. Boutami, B. Benbakir, J. Leclercq, X. Letartre, P. Regreny, M. Garrigues, P. Viktorovitch, LEOM-CNRS, École Centrale de Lyon (France); L. Legratiet, G. Beaudoin, I. Sagnes, LPNCNRS (France)

647513 Nanofluidic tuning of photonic crystal circuits [6475-40]

D. Erickson, Cornell Univ. (USA); T. Rockwood, T. Emery, A. Scherer, D. Psaltis, California Institute of Technology (USA) 
647517 Tailored nanoaggregates from functionalized organic molecules [6475-44]

M. Schiek, Univ. of Oldenburg (Germany); J. Brewer, Univ. of Southern Denmark (Denmark); F. Balzer, Univ. of Oldenburg (Germany); A. Lützen, Univ. of Bonn (Germany); K. Al-Shamery, Univ. of Oldenburg (Germany); H.-G. Rubahn, Univ. of Southern Denmark (Denmark)

647518 Microfabrication of integrated atomic vapor cells [6475-45]

D. B. Conkey, R. L. Brenning, A. R. Hawkins, Brigham Young Univ. (USA); W. Yang, B. Wu, H. Schmidt, Univ. of California, Santa Cruz (USA)

\section{POSTER SESSION}

647519 Improvement of the signal-to-noise ratio in a glass-based guided-wave optical microphone [6475-46]

H. Nikkuni, Y. Mogi, M. Hayashi, M. Ohkawa, S. Sekine, T. Sato, Niigata Univ. (Japan)

$64751 \mathrm{~A}$ Silicon p-i-n optical waveguide modulators fabricated on the silicon and silicon-oninsulator substrates [6475-47]

M.-T. Hsu, R. W. Chuang, J.-C. Liao, National Cheng Kung Univ. (Taiwan)

64751B Broadband emission of GaAs/AlGaAs quantum-well superluminescent diode at $850 \mathrm{~nm}$ [6475-48]

C. E. Dimas, C. T. Vishton, R. A. Merola, H. S. Djie, B. S. Ooi, Lehigh Univ. (USA)

64751C Image quality improved 1 X8 multimode interference coupler [6475-49]

$X$. Wang, R. T. Chen, The Univ. of Texas at Austin (USA)

Author Index 
Downloaded From: https://www.spiedigitallibrary.org/conference-proceedings-of-spie on 26 Apr 2023

Terms of Use: https://www.spiedigitallibrary.org/terms-of-use 


\title{
Conference Committee
}

\author{
Symposium Chair \\ Yakov Sidorin, Photineer Technology Group (USA) \\ Symposium Cochair
}

Ali Adibi, Georgia Institute of Technology (USA)

Program Track Chair

Yakov Sidorin, Photineer Technology Group (USA)

Conference Chairs

Yakov Sidorin, Photineer Technology Group (USA)

Christoph A. Waechter, Fraunhofer-Institut für Angewandte Optik und Feinmechanik (Germany)

Program Committee

Trevor M. Benson, The University of Nottingham (United Kingdom)

Jean-Emmanuel Broquin, École Nationale Supérieure d'Electronique et de Radioélectricité de Grenoble (France)

Venkatraman Gopalan, The Pennsylvania State University (USA)

Christoph M. Greiner, LightSmyth Technologies, Inc. (USA)

Helmut Heidrich, Fraunhofer-Institut für Nachrichtentechnik, Heinrich-HertzInstitut (Germany)

Pierre Lemaitre-Auger, École Supérieure d'Ingénieurs en Systèmes Industriels Avancés Rhôme-Alpes (France)

Christi K. Madsen, Texas A\&M University (USA)

Robert L. Nelson, Air Force Research Laboratory (USA)

Gualtiero Nunzi Conti, Istituto di Fisica Applicata Nello Carrara (Italy) and Centro Studi e Ricerche Enrico Fermi (Italy)

Session Chairs

1 Tunable WGs and WG Sensors

Jean-Emmanuel Broquin, École Nationale Supérieure d'Electronique et de Radioélectricité de Grenoble (France)

2 Glass WG Optics

Christoph A. Waechter, Fraunhofer-Institut für Angewandte Optik und Feinmechanik (Germany) 
3 Subwavelength Diffractive Photonics

Christoph M. Greiner, LightSmyth Technologies, Inc. (USA)

$4 \quad$ Modeling

Christoph A. Waechter, Fraunhofer-Institut für Angewandte Optik und Feinmechanik (Germany)

5 Recent Advances

Yakov Sidorin, Photineer Technology Group (USA)

6 Plasmonics

Gualtiero Nunzi Conti, Istituto di Fisica Applicata Nello Carrara (Italy) and Centro Studi e Ricerche Enrico Fermi (Italy)

7 Subwavelength and Microstructures

Trevor M. Benson, The University of Nottingham (United Kingdom)

$8 \quad$ PhC-based Devices

Robert L. Nelson, Air Force Research Laboratory (USA)

9 Novel Materials

Christoph A. Waechter, Fraunhofer-Institut für Angewandte Optik und Feinmechanik (Germany) 